\title{
Guideline for the identification and management of cardiometabolic risk after spinal cord injury: a case of unsubstantiated recommendations
}

\author{
Michael D. Stillman ${ }^{1} \cdot$ Steve Williams ${ }^{2}$
}

Received: 18 September 2019 / Revised: 1 October 2019 / Accepted: 3 October 2019

(c) International Spinal Cord Society 2019

\begin{abstract}
The 2018 Guideline for the Identification and Management of Cardiometabolic Risk after Spinal Cord Injury (SCI) represented the first concerted effort to address a cluster of derangements and diseases that are claiming the lives of individuals living with injuries. Its contributors and authors scoured the literature, weighed the validity, importance, and clinical relevance of what data they found, and collaborated in an effort to meaningfully improve the health and lives of people with SCI. However, we are concerned that several of the guideline's central recommendations-particularly around screening for and detection of glycemic dysregulation and dyslipidemia-have been offered prematurely. In several instances, the authors cite data from studies of people without SCI and, in our opinion, inappropriately apply those findings to support their SCI-specific suggestions. In other instances, they recommend that we employ tests whose usefulness and clinical relevance have yet to be demonstrated among people living with injuries. In short, we fear that the authors have developed clinical guidelines that are inadequately supported by data. This guideline is an extraordinary show of collaboration, and is an important first step toward understanding and treating a number of secondary cardiometabolic effects of SCI. The lack of data underpinning several of its central recommendations-making them, in our opinion, unadoptableunderscores the inadequacy of research in this area and provides a roadmap for future investigative efforts.
\end{abstract}

\section{Introduction}

We accepted this "counterpoint" assignment with trepidation. While eager to help open dialogues about what is and is not known about cardiometabolic (CM) disease in spinal cord injury (SCI), we did not want to offend the guideline [1] authors. Many of them are colleagues and friends. We hold each of them in high regard.

These authors have taken on difficult work. Even while rightly attempting to improve and standardize the care of people with SCI, they emphasize the paucity of data addressing rates of diabetes, myocardial infarction, stroke,

Michael D. Stillman

michael.stillman@jefferson.edu

1 Internal Medicine and Rehabilitation Medicine, Sydney Kimmel Medical College of Thomas Jefferson University, 1100 Walnut Street, Suite 601, Philadelphia, PA 19107, USA

2 Rehabilitation Medicine, Sydney Kimmel Medical College, Philadelphia, PA, USA and death among those with injuries. This lack of "actionable" information informs our concerns about the guideline. We respect the authors' efforts, but feel that a number of their recommendations are insufficiently supported, hence, prematurely offered.

\section{Glycemic dysregulation}

The guideline suggest that clinicians screen adults with SCI for impaired glucose tolerance (IGT) and diabetes mellitus (DM) every 3 years, and that either fasting plasma glucose (FPG), glycohemoglobin (HbA1c), or oral glucose tolerance testing (OGTT) be used. As it has never been proven that incidence of DM increases with duration of injury, the screening interval was adopted from American Diabetes Association recommendations [2] that do not specifically address SCI.

Diabetes does seem to be prevalent among people with SCI, though the scope of the problem has not been well clarified. Early work from the Veterans Administration (VA) Hospital System suggests that between 13\% and 51\% of people living with SCI are diagnosed with DM when 
undergoing OGTT [3, 4], yet in our own study of community-dwelling individuals with injuries [5], only 1 of 19 had a 2-h glucose level over $200 \mathrm{mg} / \mathrm{dL}$. Our sensethough, admittedly, unsubstantiated-is that VA data from the 1980s and 1990s may no longer be applicable, particularly with the recent emphasis on participation in exercise programs and activity-based therapies for people with SCI.

Assuming, however, that people with SCI are at risk for IGT and DM, it is important to understand the utility of the three screening tests recommended in the guideline. It is known that there is no correlation between FPG and OGTT in the setting of SCI. In Duckworth et al. and Bauman et al. participants who were diagnosed with IGT and DM during OGTT had essentially normal FPG levels [4, 6], and it is believed that the glucose excursions observed during those test were due to the loss of skeletal muscle ("sarcopenia") that ordinarily serves as a reservoir for glucose storage. People with SCI may have glucose "spikes" after mealtime in the setting of background euglycemia, hence, may "fail" OGTT while maintaining unconcerning FPG levels.

Complicating this problem is an apparent lack of correlation between $\mathrm{HbA1c}$ and OGTT results in people with SCI. In our own work [5], 9 of 19 individuals with SCI had an elevated HbA1c or 2-h glucose level, but only two had both (Table 1). One participant had an HbA1c of $5.9 \%$, diagnostic of IGT, but a 2-h glucose of $210 \mathrm{mg} / \mathrm{dL}$, indicative of DM. The other had an HbA1c of $6.1 \%$ and a 2-h glucose of $170 \mathrm{mg} / \mathrm{dL}$ - both consistent with IGT. Unpublished data from a study extension show that among 32 participants with SCI, 6 have elevated HbA1c levels, 11 have 2 -h glucose levels $\geq 140 \mathrm{mg} / \mathrm{dL}$ (indicating IGT or DM), but only two have both.

The literature comparing HbA1c with 2-h glucose levels for detection of DM is admittedly confusing. While there seem to be reliable cut-off values for both at which risk of retinopathy increases [7], several authors have shown poor correlation between the tests in specific patient populations.

Table 1 Comparison of HbA1c and OGTT among people with SCI

\begin{tabular}{lll}
\hline Subject & HbA1c & 2 h OGTT Glucose \\
\hline 1 & 5.3 & 183 \\
2 & 6.0 & 89 \\
4 & 5.3 & 182 \\
5 & 6.1 & 92 \\
6 & 5.1 & 144 \\
12 & 5.8 & 119 \\
14 & 5.9 & 210 \\
16 & 6.1 & 170 \\
17 & 5.9 & 97 \\
\hline
\end{tabular}

HbA1c 5.7-6.4 indicates IGT; $\geq 6.5$ indicates DM. 2 h OGTT 140-199 indicates IGT; $\geq 200$ indicates DM. Data from Stillman et al. [5]
In Hjellestad et al's study of people with vascular disease [8], HbA1c was only $45 \%$ sensitive in detecting DM when compared with OGTT. Picon et al. studied women previously diagnosed with gestational diabetes and found HbA1c to be only $23 \%$ sensitive [9] in diagnosing DM. Due to the unique physiology of SCI, HbA1c and OGTT may offer completely different impressions of our patients' ability to metabolize glucose. The HbA1c captures an individual's degree of glucose exposure over time, rather than reflecting the episodic excursions reflected by OGTT [7]. As people with SCI tend to have postprandial hyperglycemia but fasting euglycemia, their 2-h glucose levels may be elevated even if their HbA1c results are normal.

Given the lack of correlation between FPG, HbA1c, and OGTT among people with SCI, these three tests cannot be considered equally valid for the detection of IGT and DM in the setting of injury. As HbA1c reflects one's "overall" ability to metabolize glucose, we feel that this test is most useful to our patients. However, we suggest that prior to adopting the current guideline, we set ourselves to answer the following questions:

(1) Are postprandial glucose "spikes" seen in some people with SCI clinically significant? That is, are intermittent glucose excursions associated with microvascular consequences?

(2) Which of the three proposed screening tests best correlates with vascular complications of IGT and DM?

\section{Dyslipidemia}

The guideline suggests screening asymptomatic adults with SCI for dyslipidemia at least every three years, with more frequent testing for those with multiple cardiovascular (CV) risk factors. This recommendation is based on American Association of Clinical Endocrinologists guidelines [10] that offer individualized screening intervals for a number of patient populations but do not address special considerations in SCI.

Dyslipidemia in SCI has been well described. The primary and most common derangement is low high-density lipoprotein (HDL) levels [11], but LaFountain et al. have drawn attention to triglycerides (TG), as "adverse" values may be lower in people with SCI than in those without [12]. Despite having "benign" appearing lipid profiles, people with SCI seem to be at risk for accelerated atherosclerosis and coronary artery calcification. Both Bauman et al. and Lee et al. found high rates of positive stress tests among asymptomatic people with SCI [13, 14], and Orkazai et al. described higher coronary artery calcium scores among people with SCI than among matched noninjured controls [15]. 
Despite the fact that $\mathrm{CV}$ disease causes between $18.4 \%$ and $25 \%$ of deaths among people with SCI [16-18], no studies are available to help guide our clinical practice or our risk-reduction efforts. Nash et al. found that Niacin therapy improves the lipid profiles of people with SCI but did not follow other clinical outcomes [19]. Stillman et al's retrospective study found a $21 \%$ reduction in mortality among veterans with SCI who had been treated with HMGCoA reductase inhibitors ("statins") [20], but the work was limited by its small sample size, the homogeneity of its subjects, and the fact that it has yet to be replicated.

Contending with the difficult topic of dyslipidemia in SCI, the guideline authors suggest consideration of statin therapy with goals of reducing TG levels to $<150 \mathrm{mg} / \mathrm{dL}$ and increasing HDL levels to over $40 \mathrm{mg} / \mathrm{dL}$ in men and $50 \mathrm{mg} / \mathrm{dL}$ in women. In doing so, they cite two trials from the cardiology literature, one of which is unconvincing and one of which offers results that are not easily applicable to people with SCI.

HOPE-3 was a placebo-controlled trial investigating statin use in people with risk factors for $\mathrm{CV}$ disease but without elevated low-density lipoprotein (LDL) levels [21]. With just over 5 years of follow up, statin therapy yielded a $1.1 \%$ absolute risk reduction (ARR) in the combined endpoint of death from $\mathrm{CV}$ causes, nonfatal myocardial infarction, and nonfatal stroke. However, the number of participants needed to treat to prevent any one of these coprimary outcomes was 91 , and among subjects taking statins, there was no reduction in overall mortality, CV-related mortality, resuscitated cardiac arrest, or angina with evidence of ischemia. These results seem far less convincing than those from foundational cardiology studies that showed up to an ARR of $3.1 \%$ (25\% relative risk reduction) in major adverse coronary events (MACE) among people with known CV disease or DM taking statins [22].

In the Jupiter Trial, people with normal LDL but elevated C-reactive protein (CRP) levels were assigned to take either rosuvastatin or placebo [23]. The study was stopped after $<2$ years, as those in the active treatment group had significant reductions in a number of outcomes including myocardial infarction, stroke, and unstable angina. While it is tempting to apply these findings to people with SCIparticularly as they tend to have higher CRP levels than do matched non-injured controls [24]—CRP may be affected by a number of factors including level of injury, decreased mobility, urinary catheter use, and recent infection [25-27]. There is simply no way to tease out whether an elevated CRP value in someone with SCI is due to endovascular inflammation or to the injury, itself.

Given the persistent uncertainty over which screening tests and pharmacological interventions may help reduce CV morbidity and mortality among people with SCI, the following questions ought to be answered prior to adoption of this guideline:

(1) Is Stillman et al's study of veterans with SCI reproducible? That is, can we demonstrate in a larger and more diverse subject pool-even retrospectively - that statin therapy significantly reduces mortality among people with SCI?

(2) Are there widely available and affordable tests that can predict large vessel atherosclerotic disease in SCI? Endothelial dysfunction (EDx) is found in people without SCI in the earliest stages of coronary artery disease (CAD) [28] and has been shown to predict MACE [29-31]. Are there inflammatory markers or office or laboratory-based tests for EDx that can reliably predict CAD and MACE among people with SCI and, hence, help direct preventive therapy to those most at risk?

(3) Does autonomic dysfunction-particularly autonomic dysreflexia-cause MACE among people with SCI? If so, what are we to do about that?

\section{Conclusion}

The guideline authors took on a worthy and ambitious project. In their introduction, they shared with readers which questions they had set out to address, and many of them focused on understanding the incidence of CV complications of SCI, clarifying which screening tests for CM disease are most accurate among people with SCI, and which interventions improve health outcomes. The fact that the Guideline failed to answer most of these questions speaks to an alarming paucity of evidence rather than lack of effort or vision by the authors. We feel that this Guideline was prematurely published and that some of its recommendations ought not to be adopted by clinicians caring for people with SCI. However, we feel with equal conviction that its authors have done a great service to our professional community and to individuals with SCI. In publicly grappling with what is and is not known about CM disease in SCI, they have both laid out an ambitious research agenda and challenged us all to do better. And, in this way, the document is invaluable.

\section{Compliance with ethical standards}

Conflict of interest The authors declare that they have no conflict of interest.

Publisher's note Springer Nature remains neutral with regard to jurisdictional claims in published maps and institutional affiliations. 


\section{References}

1. Nash MS, Groah SL, Gater D, Dyson-Hudson TA, Lieberman JA, Myers J. et al. Identification and Management of Cardiometabolic Risk after Spinal Cord Injury: Clinical Practice Guideline for Health Care Providers. Topics Spinal Cord Injury Rehabil. 2018;24:379-423.

2. Marathe PH, Gao HX, Close KL. American diabetes association standards of medical care in diabetes 2017. J Diabetes. 2017;9:320-4.

3. Bauman WA, Adkins RH, Spungen AM, Waters RL. The effect of residual neurological deficit on oral glucose tolerance in persons with chronic spinal cord injury. Spinal Cord. 1999;37:765-71.

4. Duckworth WC, Solomon SS, Jallepalli P, Heckemeyer C, Finnern J, Powers A. Glucose intolerance due to insulin resistance in patients with spinal cord injuries. Diabetes. 1980;29:906-10.

5. Stillman M, Graves D, Lenneman C, Williams S. Neurogenic bowel, disordered glycemic control and chronic spinal cord injury: a preliminary investigation. Phys Med Rehabilitation - Int. 2017:4:1-3.

6. Bauman WA, Spungen AM. Disorders of carbohydrate and lipid metabolism in veterans with paraplegia or quadriplegia: a model of premature aging. Metabolism. 1994;43:749-56.

7. International Expert Committee. International Expert Committee report on the role of the A1C assay in the diagnosis of diabetes. Diabetes Care. 2009;32:1327-34.

8. Hjellestad ID, Astor MC, Nilsen RM, Softeland E, Jonung T. HbA (1)c versus oral glucose tolerance test as a method to diagnose diabetes mellitus in vascular surgery patients. Cardiovasc Diabetol. 2013;12:79-2840-12-79.

9. Picon MJ, Murri M, Munoz A, Fernandez-Garcia JC, GomezHuelgas R, Tinahones FJ. Hemoglobin A1c versus oral glucose tolerance test in postpartum diabetes screening. Diabetes Care. 2012;35:1648-53.

10. Jellinger PS, Handelsman Y, Rosenblit PD, Bloomgarden ZT, Fonseca VA, Garber AJ, et al. American Association of Clinical Endocrinologists and American College of Endocrinology guidelines for management of dyslipidemia and prevention of cardiovascular disease. Endocr Pr. 2017;23(Suppl 2):1-87.

11. Bauman WA, Spungen AM, Zhong YG, Rothstein JL, Petry C, Gordon SK. Depressed serum high density lipoprotein cholesterol levels in veterans with spinal cord injury. Paraplegia. 1992; 30:697-703.

12. La Fountaine MF, Cirnigliaro CM, Hobson JC, Dyson-Hudson TA, Mc Kenna C, Kirshblum SC, et al. Establishing a threshold to predict risk of cardiovascular disease from the serum triglyceride and high-density lipoprotein concentrations in persons with spinal cord injury. Spinal Cord. 2018;56:1051-8.

13. Bauman WA, Raza M, Spungen AM, Machac J. Cardiac stress testing with thallium-201 imaging reveals silent ischemia in individuals with paraplegia. Arch Phys Med Rehabil. 1994;75:946-50.

14. Lee CS, Lu YH, Lee ST, Lin CC, Ding HJ. Evaluating the prevalence of silent coronary artery disease in asymptomatic patients with spinal cord injury. Int Heart J. 2006;47:325-30.

15. Orakzai SH, Orakzai RH, Ahmadi N, Agrawal N, Bauman WA, Yee $\mathrm{F}$, et al. Measurement of coronary artery calcification by electron beam computerized tomography in persons with chronic spinal cord injury: evidence for increased atherosclerotic burden. Spinal Cord. 2007;45:775-9.
16. National Spinal Cord Injury Statistical Center. Spinal cord injury facts and figures at a glance. Birmingham: University of Alabama at Birmingham; 2016. www.nscisc.uab.edu. Accessed 16 Dec 2017.

17. Garshick E, Kelley A, Cohen SA, Garrison A, Tun CG, Gagnon $\mathrm{D}$, et al. A prospective assessment of mortality in chronic spinal cord injury. Spinal Cord. 2005;43:408-16.

18. Rabadi MH, Mayanna SK, Vincent AS. Predictors of mortality in veterans with traumatic spinal cord injury. Spinal Cord. 2013;51:784-8.

19. Nash MS, Lewis JE, Dyson-Hudson TA, Szlachcic Y, Yee F, Mendez AJ, et al. Safety, tolerance, and efficacy of extendedrelease niacin monotherapy for treating dyslipidemia risks in persons with chronic tetraplegia: a randomized multicenter controlled trial. Arch Phys Med Rehabil. 2011;92:399-410.

20. Stillman MD, Aston CE, Rabadi MH. Mortality benefit of statin use in traumatic spinal cord injury: a retrospective analysis. Spinal Cord. 2016;54:298-302.

21. Yusuf S, Bosch J, Dagenais G, Zhu J, Xavier D, Liu L, et al. Cholesterol lowering in intermediate-risk persons without cardiovascular disease. N Engl J Med. 2016;374:2021-31.

22. Heart Protection Study Collaborative Group. MRC/BHF heart protection study of cholesterol lowering with simvastatin in 20,536 high-risk individuals: a randomised placebo-controlled trial. Lancet. 2002;360:7-22.

23. Ridker PM, Danielson E, Fonseca FA, Genest J, Gotto AM Jr, Kastelein JJ, et al. Rosuvastatin to prevent vascular events in men and women with elevated C-reactive protein. N Engl J Med. 2008;359:2195-207.

24. Liang H, Mojtahedi MC, Chen D, Braunschweig CL. Elevated Creactive protein associated with decreased high-density lipoprotein cholesterol in men with spinal cord injury. Arch Phys Med Rehabil. 2008;89:36-41.

25. Goldstein RL, Walia P, Teylan M, Lazzari AA, Tun CG, Hart JE, et al. Clinical factors associated with C-reactive protein in chronic spinal cord injury. Spinal Cord. 2017;55:1088-95.

26. Gibson AE, Buchholz AC, Martin Ginis KA, SHAPE-SCI Research Group. C-Reactive protein in adults with chronic spinal cord injury: increased chronic inflammation in tetraplegia vs paraplegia. Spinal Cord. 2008;46:616-21.

27. Morse LR, Stolzmann K, Nguyen HP, Jain NB, Zayac C, Gagnon $\mathrm{DR}$, et al. Association between mobility mode and C-reactive protein levels in men with chronic spinal cord injury. Arch Phys Med Rehabil. 2008;89:726-31.

28. Ross R. Atherosclerosis is an inflammatory disease. Am Heart J. 1999;138:S419-20.

29. Celermajer DS, Sorensen KE, Bull C, Robinson J, Deanfield JE. Endothelium-dependent dilation in the systemic arteries of asymptomatic subjects relates to coronary risk factors and their interaction. J Am Coll Cardiol. 1994;24:1468-74.

30. Yeboah J, Crouse JR, Hsu FC, Burke GL, Herrington DM. Brachial flow-mediated dilation predicts incident cardiovascular events in older adults: the Cardiovascular Health Study. Circulation. 2007;115:2390-7.

31. Yeboah J, Folsom AR, Burke GL, Johnson C, Polak JF, Post W, et al. Predictive value of brachial flow-mediated dilation for incident cardiovascular events in a population-based study: the multi-ethnic study of atherosclerosis. Circulation. 2009;120: 502-9. 